\title{
GENETIC AND PHYSIOLOGICAL CHARACTERISATION OF PHENOL- AND $P$-CRESOL-DEGRADING BACTERIA SELECTED FOR BIOAUGMENTATION IN OIL- AND PHENOL-POLLUTED AREA
}

\author{
Merike Merimaa \\ Eeva Heinaru \\ Merit Lehiste \\ Signe Viggor \\ Ain Heinaru \\ Tartu University, Estonia
}

\begin{abstract}
Successful bioaugmentation requires that bacterial strains introduced into the polluted area must be able to adapt to new environmental conditions and retain high enough catabolic activity. The strains should degrade pollutant present at high concentrations, while having high affinity for the pollutants for their thorough degradation. The ransfer of genetic information from introduced donor strain to indigenous bacterial population increases the biodegradation potential. As laboratory-selected strains can be poor survivors and lose catabolic activity in mixed microbial ecosystems, the indigenous biodegradative strains isolated from the river water continuously polluted with phenolic compounds of oil shale leachate may serve as inoculants for bioaugmentation. We have shown that the native phenol- and $p$-cresol-degrading community could be grouped according to the presence of catabolic genes involved in catabolism of aromatic compounds. The selected representative strains of different catabolic types of degradation of phenol and p-cresol were identified as Pseudomonas mendocina (strain $\mathrm{PCl}$ ) and $P$. fluorescens (strains PC18, PC24). Catabolic potential of these strains was studied on the basis of phenol hydroxylase, $p$-cresol methylhydroxylase and catechol 2,3-dioxygenase genes. The occurrence and conjugation of plasmid DNA were revealed in these strains. The ability of the selected strains to degrade several phenolic compounds in natural phenolic wastewater in which the compounds were present in multicomponent mixtures, was investigated through laboratory microcosm studies. To elucidate the extent of interactions among the used bacterial strains single and mixed cultures were set up. The biodegradation activity of strains in microcosms was examined through viable counts, consumption of phenolic compounds and detecting the presence of catabolic genes by hybridization. During the experiments ( 30 days) the introduced bacteria remained viable
\end{abstract}


KALMAR ECO-TECH'03

Bioremediation and Leachate Treatment

KALMAR, SWEDEN, November 25-27, 2003

even when the substrates were depleted. The mixture of strains was more effective in the decomposition of phenolic compounds from the natural wastewater as compared with the single culture conditions and the metabolic activity and cell density of each strain were co-ordinated within a specific time scale.

The behaviour of strains in the phenolic leachate depended on the growth kinetics of the strains $\left(K_{s}, \mu\right)$.

\section{KEYWORDS}

Biodegradation; Catabolic genes; Growth kinetics; Microcosms; Oil shale leachate; Phenolic compounds

\section{INTRODUCTION}

During the last century industrial and agricultural activities have resulted in the release of large quantities of toxic xenobiotic (i.e., man-made) compounds into diverse ecosystems [1]. Likewise, oil shale industry has created serious pollution problem in the north-eastern part of Estonia. The main environmental damage has been caused by phenolic leachate formed from industrial water used for compacting of ash and precipitation.

Bioremediation of contaminated environments by microorganisms has become an accepted technology for the restoration of polluted environments [2]. Naturally occurring microbial activities have always been the starting point for all biotechnological applications [3]. It is important to understand the bioavailability of a pollutant, the survival and catabolic activity of added microorganisms. Naturally evolved microorganisms tend to be more competitive in degrading different pollutants since they have already survived natural selection. It is therefore necessary to isolate, identify and characterise pollutant-degrading bacterial strains for analysing their in situ activity. Important progress in the elucidation of individual metabolic pathways has been gained, but little is known about the pathways operating in natural communities, where extensive sharing of nutritional resources is common [4]. In addition, the interactions between microbial species in a mixed culture conditions at decomposition of a mixture of chemical compounds should be important to understand the interactions among undergoing biodegradation.

The results of our previous work demonstrate multiplicity of catabolic types of degradation of phenol and $p$-cresol and the existence of characteristic assemblages of species and specific genotypes among the strains isolated from river water continuously polluted with phenolic compounds of oil shale leachate [4]. In this work three main 
KALMAR ECO-TECH'03

Bioremediation and Leachate Treatment

KALMAR, SWEDEN, November 25-27, 2003

catabolic types of degradation of phenol and $p$-cresol were revealed. We found that sequential degradation of substrates and diauxic growth is caused by reversible accumulation of the intermediates [5]. It has already been shown that the mixed culture of strains with different catabolic types made it possible to overcome the incompatibilities at degradation of substrate mixtures [6]. The main goal of this paper was to investigate the biodegradation potential and kinetics of isolated bacteria potentially useful for bioaugmentation experiments of phenol polluted wastewaters.

\section{MATERIALS AND METHODS}

\subsection{Bacterial strains, medium and culture conditions}

Bacterial strains used in this study were Pseudomonas mendocina PC1, Pseudomonas fluorescens C PC24, $P$. fluorescens G PC18. These strains are representatives of different catabolic types and they degrade phenol and p-cresol via meta-meta, ortho-ortho, metaortho and meta-meta pathways, respectively [4]. Reference strains as the sources of catechol 2,3-dioxygenase and phenol monooxygenase genes were P. putida PaW160 and P. putida EST 1412, respectively.

Pure cultures were stored in $30 \%$ glycerol at $-708 \mathrm{C}$ or incubated in agar plates with minimal media containing M9 salts [7] and trace elements [8] supplemented with phenol $(2.5 \mathrm{mM})$.

\subsection{Oil shale leachate microcosms}

Microcosms experiments were performed with single strains (PC1, PC18, PC24) and mixed culture of these strains in natural phenolic wastewater ( $\mathrm{pH} \mathrm{12)}$. $\mathrm{pH}$ was conducted to 7,5 with $\mathrm{HCl}$ and $\mathrm{M} 9$. Different microcosm cultures were grown in $150 \mathrm{ml}$ Erlenmeyer flasks containing $50 \mathrm{ml}$ filter-sterilised oil shale leachate $(0,22 \mu \mathrm{m}$ pore size Millipore filter). The concentrations of phenolic compounds in the media were ( $\mathrm{mg} \mathrm{l}^{-}$ ${ }^{1}$ ):phenol - 136.1; $p$-cresol - 25.8; o-cresol - 9.5; 2,3-dimethylphenol - 8.33; 2,4dimethylphenol - 4.31; 2,6-dimethylphenol - 3.85; 3,4-dimethylphenol (3,4-DMP) - 1.63; 3,5-dimethylphenol - 4.75. Microcosms were inoculated with cells pregrown on phenol at an initial cell concentration of $0.5-1 \times 10^{7} \mathrm{CFU} \mathrm{ml} l^{-1}$ and were grown on a rotary shaker $(180 \mathrm{rpm})$ at $308 \mathrm{C}$. Abiotic control variant was not inoculated. After inoculation, samples for substrate analysis were periodically taken, filtered through $0.22 \mu \mathrm{m}$ Millipore filter and stored frozen until analysis with HPLC. For the enumeration of bacteria, serial dilutions of samples in $0.9 \% \mathrm{NaCl}$ were plated out onto phenol $(2.5 \mathrm{mM})$ minimal medium in triplicate. Counts were obtained after 5 days of incubation at $308 \mathrm{C}$. 


\subsection{HPLC analyses}

High-performance liquid chromotography (HPLC) analyses of phenolic compounds were performed using a Kontron Instruments HPLC system 500 (BIO-TEK $®$ Instruments, Inc.) with an automatic injector (Autosampler 560), a binary pump with high pressure gradient mixer (Pump System 525) and an UV diode array detector (diode Array Detector 540). Separation of phenolic compounds was achieved using a Hypersil ODS (250x4.6 $\mathrm{mm}$ i.d., $5 \mu \mathrm{m})$ analytical column and a Supersher ${ }^{\circledR} 100$ RP-18 (10x12 mm i.d., $\left.4 \mu \mathrm{m}\right)$ pre-column from Hewlett-Packard (Germany). Elution was carried out at a flow rate of 1 $\mathrm{ml} \mathrm{min}{ }^{-1}$ with the mobile phase $0.005 \mathrm{M}$ aqueous phosphoric acid-acetonitrile (64:36). Separation was carried out at room temperature. The volume of sample injected by automatic injection was $20 \mu \mathrm{l}$. Compounds were performed comparing UV spectra and retention times with those of reference substrates. Substrates were quantified by calibration with standards. For the registration and calculations of HPLC data the programme KromaSystem2000 was used.

\subsection{Monitoring the single strain from mixed culture}

Cells from colonies grown on minimal medium with phenol were transferred with sterile toothpick onto the uncharged nylon membranes Hybond-N (Amersham Pharmacia Biotech. Inc.), then placed onto Luria-Bertani agar media followed by incubation at $308 \mathrm{C}$ for $24 \mathrm{~h}$. Cell lysis was achieved after $7 \mathrm{~min}$ in $0.5 \mathrm{M} \mathrm{NaOH}, 1.5 \mathrm{M} \mathrm{NaCl}$, and subsequent neutralisation two times for 3 min each in $0.5 \mathrm{M}$ Tris- $\mathrm{HCl}, 1.5 \mathrm{M} \mathrm{NaCl}, \mathrm{pH}$ 7.2. Membranes were washed in $2 x$ SSC ( $1 \times$ SSC is $0.15 \mathrm{M} \mathrm{NaCl}$ and $0.015 \mathrm{M}$ sodium citrate, $\mathrm{pH}$ 7.0) and air dried. After the crosslinking of DNA, the membranes were hybridised with radioactively labelled gene probes, obtained by PCR as 356-bp $x y l E$ gene products from strains $\mathrm{PC} 1$ and $\mathrm{PC} 18$ and 947-bp pheA gene product from strain PC24 [4]. The hybridisation procedure was performed in accordance with the manufacturer's protocol. In the screening experiments, growth on Kings B agar (Merck, Darmstadt, Germany) was used as an additional test for fluorescent Pseudomonas strains (PC18 and PC24). The colonies with catechol 2,3-dioxygenase activity were tested with $0.5 \%$ catechol (strains $\mathrm{PC} 1$ and $\mathrm{PC} 18)$.

\subsection{Determination of biodegradation kinetic parameters}

The kinetic parameters of growth on phenol $(2.5 \mathrm{mM})$ and $p$-cresol $(1.3 \mathrm{mM})$ were derived from batch cultivation experiments in the exponential growth phase. The phenoland $p$-cresol-degrading activities for $K_{s}$ (the half-saturation constant) assay were calculated from a phenol and $p$-cresol oxygenation-dependent-consumption rate. For determining the specific growth rate $(\mu)$, cell density of batch cultures of strains was monitored spectrophotometrically by measuring the absorbance at a wavelength of 580 $\mathrm{nm}$. For each batch culture, the specific growth rate was estimated from the slope of semilogarithmic plots of optical density against cultivation time in the exponential growth phase, where $\mu$ had approximately constant value. 


\subsection{Microcosms}

Decomposition of phenolic compounds in oil shale leachate microcosms conditions by single and mixed culture of different bacterial strains is shown in Figure 1. There were no changes in the concentrations of phenolic compounds revealed during 30 days in the abiotic control variant. The strain PC18 differed from others by its inability to catabolise $p$-cresol and $o$-cresol during the first 15 days (see Figure 1. A and C). In contrast, 3,4dimethylphenol was completely degraded during the first day by the same strain (see Figure 1. D). The incomplete disappearance of 3,4-dimethylphenol was revealed in case of the strains PC1 and PC24 and $o$-cresol by strain PC24. All three strains investigated decomposed completely phenol and $p$-cresol.

The mixture of the strains PC1, PC1 8 and PC24 was more effective in the decomposition of phenolic compounds from leachate as compared with the single culture conditions. This was most remarkable when studying the decomposition processes within specific time scale (see Figure 1). However, after loner period of incubation the single strains were mostly as effective as the mixture of the strains. For example, after 15 days, the strain PC18 appeared to be as effective in decomposing each phenolic compound studied as compared to the mixture of the strains.

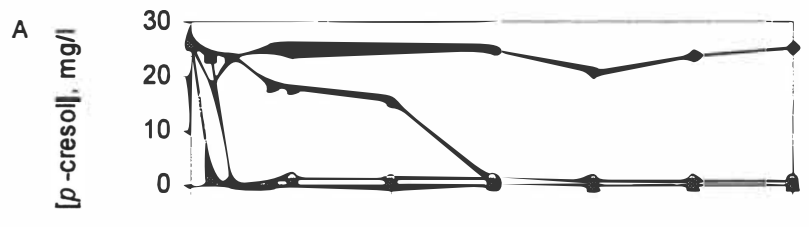

B
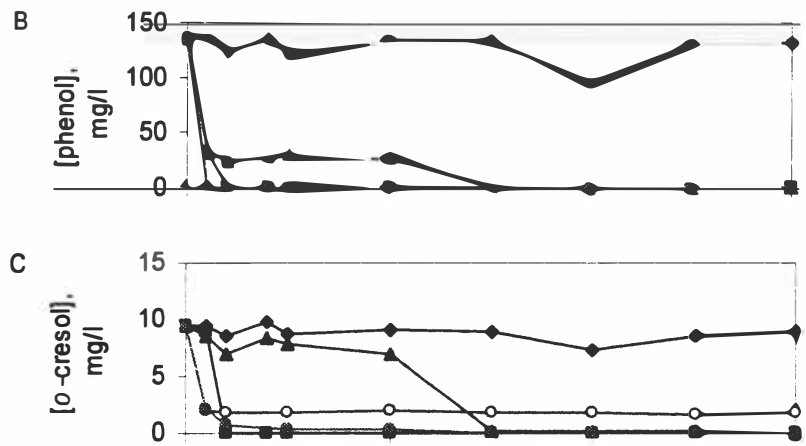
KALMAR ECO-TECH'03

Bioremediation and Leachate Treatment

KALMAR, SWEDEN, November 25-27, 2003
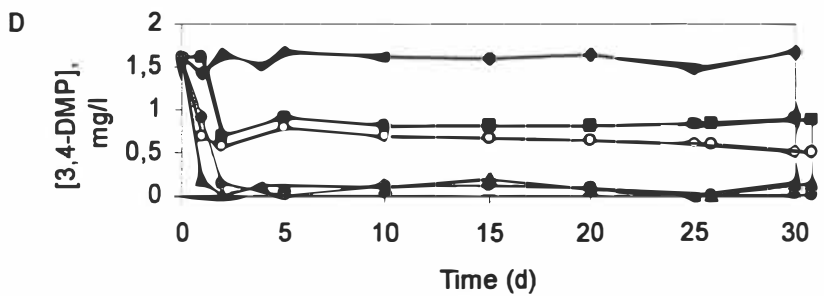

$\multimap \mathrm{R}-\multimap \mathrm{PC} 1 \multimap \mathrm{PC} 18 \multimap \mathrm{PC} 24 \rightarrow \mathrm{M}$

Figure 1. Degradation of phenolic compounds in oil shale leachate by single strains (PC1, PC18 and PC24) and mixed culture (M). $R$ - abiotic control variant.

It was shown that the numbers of CFU per ml of strains PC1 and PC18 in single culture and CFU per ml of mixed culture increased during the first day of incubation and after period of stabilisation after the day 5 the density remained constant (see Figure 2).

The three strains, $\mathrm{PC} 1, \mathrm{PC} 18$ and $\mathrm{PC} 24$ were representatives of the three catabolic types revealed among numerous indigenous strains isolated from the wastewater of the oil shale leachate. The bacterial population in this wastewater had been under natural selection against phenolic pollution for a number of years. It is reasonable to suppose that these bacteria evolved in natural conditions and the traits present in the isolated strains reflected its role in the microbial community in the decomposition of pollutants during natural bioremediation. Indeed, the ratio between the numbers of cells of each strain in mixed population was different during the decomposition process (see Figure 3). The role of strain PC1, having only the active meta pathway, was bigger in conditions with higher concentrations of phenolic compounds. The strain PC24 contains ortho pathway and therefore mostly involved in the degradation of phenol. The strain PC18 was the most interesting one. It could have been evolved to decompose dimethylphenols in the presence of phenol and $p$-cresol. It is well known that meta and ortho ring fission of phenolic compounds are alternative pathways. In strain PC18 phenol induces the $p$-cresol protocatechuate pathway which is not needed for the catabolism of phenol itself [4]. We suppose that in the presence of dimethylphenols which are more toxic phenolic compounds comparing with phenol and $p$-cresol, the induction of protocatechuate pathway favours degradation of dimethylphenols. 


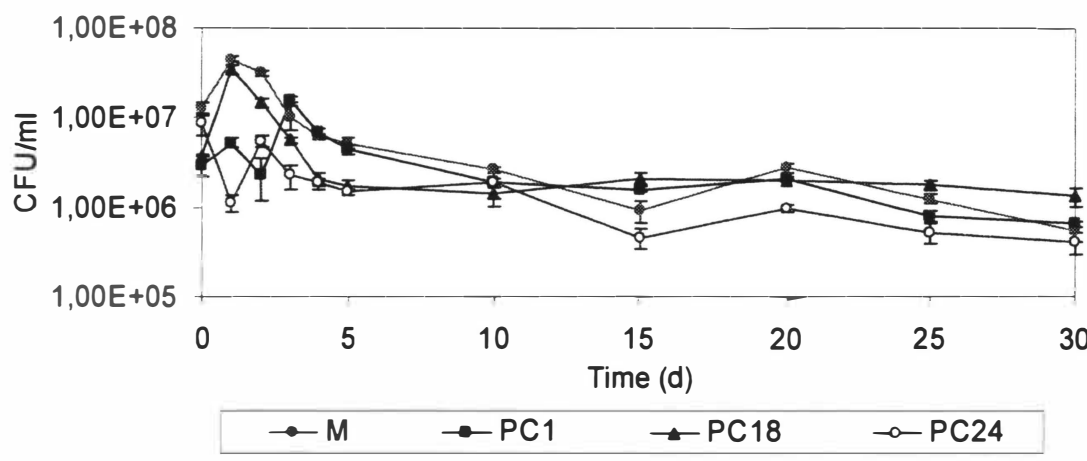

Figure 2. Numbers of CFU per ml of the bacterial populations in single and mixed culture conditions.

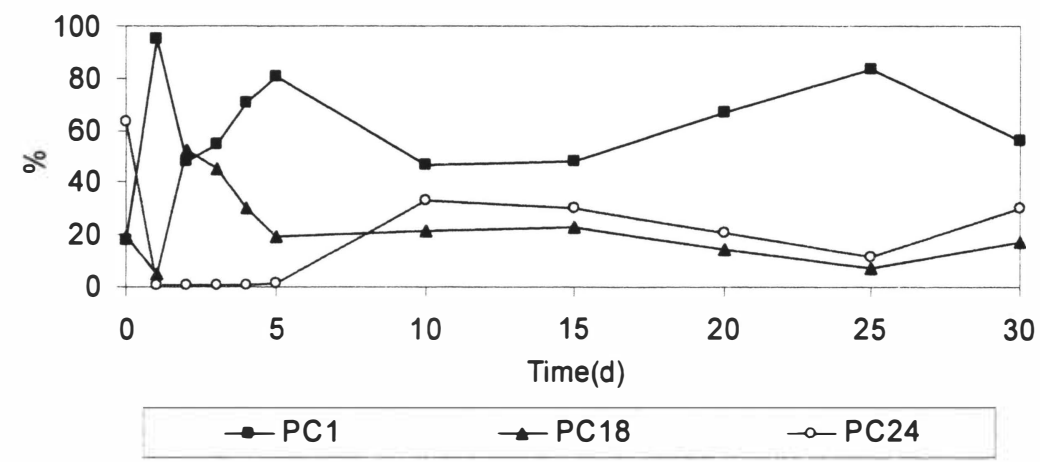

Figure 3. Proportion between separate strains in bacterial population during the 30-days incubation period in mixed culture. The relative percentage of each strain is derived from the sum total of the individual strains.

\subsection{Kinetic analyses}

The kinetic constants, specific growth rate $\mu$ and half saturation coefficient $K_{s}$ affect the biodegradation of a compound. We suppose that they must collectively be reflected in relative biodegradation rates. It can be seen from the data of the kinetic constants for the phenol- and $p$-cresol-oxygenating activities of the strains PC1, PC18 and PC24, presented in Table 1, that there is almost a two-fold variation in the $\mu$ values during the growth of different strains on phenol. Less variation was detected in the $\mu$ values during the growth of these three strains on $p$-cresol. High values of $\mu$ of strain $\mathrm{PCl}$ during the growth on 
KALMAR ECO-TECH'03

Bioremediation and Leachate Treatment

KALMAR, SWEDEN, November 25-27, 2003

both phenol and $p$-cresol reflected a good potential of this strain in rapid degradation of these compounds. The $K_{s}$ values for phenol and $p$-cresol showed relatively wide variation. The lowest $K_{s}$ values were lowest for phenol and $p$-cresol in strain PC18 and the highest for the strain PCl. The low $K_{s}$ values indicate the highest affinity of strain PC18 to both substrates and, therefore, the ability of this strain to grow at low substrate concentrations.

Table 1. The kinetic constants for the phenol-and p-cresol-oxygenating activity of strains PC1, PC18 and PC24.

\begin{tabular}{ccccc}
\hline Strain & \multicolumn{2}{c}{ Phenol } & \multicolumn{2}{c}{$p$-cresol } \\
\cline { 2 - 5 } & $\mathrm{K}_{\mathrm{s}}(\mu \mathrm{M})$ & $\mu\left(\mathrm{h}^{-1}\right)$ & $\mathrm{K}_{\mathrm{s}}(\mu \mathrm{M})$ & $\mu\left(\mathrm{h}^{-1}\right)$ \\
\hline PC1 & $0,404 \pm 0,084$ & $0,83 \pm 0,17$ & $0,129 \pm 0,041$ & $0,81 \pm 0,12$ \\
\hline PC18 & $0,038 \pm 0,003$ & $0,42 \pm 0,04$ & $0,015 \pm 0,003$ & $0,67 \pm 0,10$ \\
\hline PC24 & $0,127 \pm 0,045$ & $0,75 \pm 0,11$ & $0,040 \pm 0,006$ & $0,68 \pm 0,06$ \\
\hline
\end{tabular}

Data were obtained from at least three separate and independent experiments

\section{CONCLUSION}

- The mixture of the strain P. mendocina PC1 and P. fluorescns strains PC18 and PC24 was more effective on the decomposition of phenolic compounds in natural wastewaters.

- The ratio between the numbers of cells of the strains PC1, PC18 and PC24 in mixed population was different during the decomposition process. The role of the strain PCl, having only active meta pathway, was more important in conditions with higher concentrations of phenolic compounds. The strain PC24 contains only ortho pathway and the role of this strain can be combined mostly with the degradation of phenol. The main role of the strain PC18 was the degradation of dimethylphenols.

- Comparison of kinetic parameters (specific growth rate - $\mu$ and half saturation constant $-K_{s}$ ) reflected the differences in biodegradation rates in mixed populations.

\section{ACKNOWLEDMENTS}

The study was funded by the Estonian Science grant No 4344 .

\section{REFERENCES}

[1] Top, E. M., Springael, D., Boon, N., 2002. Catabolic mobile genetic elements and their potential use in bioaugmentation of polluted soils and water. FEMS Microbiol. Ecol., 42: 199-208. DOI: https://doi.org/10.1016/S0168-6496(02)00370-7 
KALMAR ECO-TECH '03

Bioremediation and Leachate Treatment

KALMAR, SWEDEN, November 25-27, 2003

[2] Wikstrom, P., Hagglund, L., Forsman, M., 2000. Structure of a natural microbial community in a nitroaromatic contaminated groundwater is altered during biodegradation of extrinsic, but not intrinsic substrates. Microb. Ecol., 39: 203-210.

DOI: https://doi.org/10.1007/s002480000001

[3] Pieper, D. H., Reineke, W., 2000. Engineering bacteria for bioremediation. Curr. Opin. Biotechnol.,1 1: 262-270 DOI: https://doi.org/10.1016/S0958-1669(00)00094-X

[4] Heinaru, E., Truu, J., Stottmeister, U., Heinaru, A., 2000. Three types of phenol and $p$-cresol catabolism on phenol- and $p$-cresol - degrading bacteria isolated from river water continuously polluted with phenolic compounds. FEMS Microbiol. Ecol., 31: 195-205. DOI: https://doi.org/10.1111/j.1574-6941.2000.tb00684.x

[5] Heinaru, E., Viggor, S., Vedler, E., Truu J., Merimaa, M., Heinaru, A., 2001. Reversible accumulation of $p$-hydroxybenzoate and catechol determines the sequential decomposition of phenolic compound in mixed substrate cultivations in pseudomonads. FEMS Microbiol. Ecol., 37: 79-89.

DOI: https://doi.org/10.1111/j.1574-6941.2001.tb00855.x

[6] Viggor, S., Heinaru, E., Loponen, J., Merimaa, M., Tenno, T., Heinaru, A., 2002. Bioderadation of dimethylphenols by bacteria with different ring-cleavage pathways of phenolic compounds. Environ. Sci. Res., 1: 19-26.

DOI: https://doi.org/10.1007/BF02987421

[7] Adams, M.H., 1959. Bacteriophages. pp 445-468. Interscience Publiscers, New York.

[8] Bauchop, T., Elsden, S.R., 1960. The growth of microorganisms in relation to their energy supply. J. Gen. Microbiol., 64: 4353-4356. 